DOI: https://doi.org/10.46296/rc.v4i8edespdic.0031

\title{
La tributación y eficiencia administrativa en las pequeñas y medianas empresas (PYMES), registradas en el MIPRO del cantón \\ Manta, provincia de Manabí
}

\section{Taxation and administrative efficiency in small and medium-sized enterprises (SMEs), registered in the MIPRO of the Manta canton, province of Manabí}

\author{
Tómala-Pinto Jesús Jacinto \\ Ing. Comercial, MBA en Administración de Empresas. \\ Profesor titular de la Facultad de Contabilidad y Auditoría, \\ Universidad Laica Eloy Alfaro de Manabí, ULEAM. Manta, Ecuador. \\ Correo: jesustomala@yahoo.es \\ Zambrano-Loor Rita Mayda \\ Ing. en Contabilidad y Auditoría, Magister en Tributación y Finanzas. \\ Profesora titular de la Facultad de Contabilidad y Auditoría, \\ Universidad Laica Eloy Alfaro de Manabí, ULEAM. Manta, Ecuador. \\ Correo: ritazambranoloor@gmail.com

\section{Rivas-Cedeño Limber Leónidas} \\ Abogado de los Juzgados de la República del Ecuador. \\ Profesor titular de la Facultad de Contabilidad y Auditoría, \\ Universidad Laica Eloy Alfaro de Manabí, ULEAM. Manta, Ecuador. \\ Correo: limber1962@hotmail.es
}

\begin{abstract}
RESUMEN
Los impuestos proporcionan al gobierno fondos para invertir en el desarrollo, aliviar la pobreza, proporcionar servicios públicos y construir infraestructura material y social para el crecimiento a largo plazo. Además, existe un vínculo mutuamente beneficioso entre la fiscalidad y la buena gobernanza. De esta manera, la innovación en la asistencia tributaria y para el desarrollo puede fortalecer el sistema tributario, ya que los impuestos pueden tener un impacto positivo en la calidad de la gobernanza y la relación entre el gobierno y los ciudadanos. A su vez, la buena gobernanza puede tener un impacto positivo en el cumplimiento y la movilización de ingresos. El objetivo de esta investigación fue determinar la tributación y eficiencia administrativa en las pequeñas y medianas empresas (pymes), registradas en el MIPRO del cantón Manta, provincia de Manabí. Para ello se recurrió a entrevistar a las pymes del sector, al igual que a la comunidad universitaria de la Facultad de Contabilidad y Auditoría. A través de esta investigación fue posible lograr el acercamiento y la cooperación entre la Facultad de Contabilidad y Auditoría, Universidad - Pequeñas y Medianas Empresas.
\end{abstract}

Palabras claves: Pymes, Tributación, Eficiencia administrativa, Cooperación.

Información del manuscrito:

Fecha de recepción: 06 de octubre de 2021.

Fecha de aceptación: 30 de noviembre de 2021.

Fecha de publicación: 01 de diciembre de 2021. 


\begin{abstract}
Taxes provide the government with funds to invest in development, alleviate poverty, provide public services, and build physical and social infrastructure for long-term growth. Furthermore, there is a mutually beneficial link between taxation and good governance. In this way, innovation in tax and development assistance can strengthen the tax system, as taxes can have a positive impact on the quality of governance and the relationship between government and citizens. In turn, good governance can have a positive impact on compliance and revenue mobilization. The objective of this research was to determine the taxation and administrative efficiency in small and medium-sized companies (SMEs), registered in the Mipro of the Manta canton, Manabí province. To do this, they resorted to interviewing SMEs in the sector, as well as the university community of the Faculty of Accounting and Auditing. Through this research it was possible to achieve rapprochement and cooperation between the Faculty of Accounting and Auditing, University Small and Medium Enterprises.
\end{abstract}

Keywords: SMEs, Taxation, Administrative efficiency, Cooperation.

\title{
1. INTRODUCCIÓN
}

En la situación actual, es evidente la relación entre los diferentes indicadores de sostenibilidad que brindan las múltiples encuestas basadas en la viabilidad del desarrollo y la innovación tributaria. Como resultado, Viktor Et al., (2016) identificaron las diferentes características de sostenibilidad de las pymes y los departamentos tributarios. Sin embargo, no existe un equilibrio entre las diferentes áreas de la sostenibilidad, y concluyeron que es necesario promover un enfoque inclusivo para promover prácticas de gestión de la sostenibilidad en la industria de las microfinanzas. En este caso, para optimizar el desempeño en sustentabilidad del primer grupo de interés compuesto principalmente por bancos, es necesario impulsar reformas y acciones sociales y de gobernanza: identificar mercados objetivo, establecer metas de desarrollo y revisar el posicionamiento social del directorio, o políticas y procedimientos de recursos humanos relacionados con la protección o seguridad social.

A gran escala, la gran cantidad de activos y la gran cantidad de oficinas son indicadores importantes para caracterizar los diferentes grupos, pero no tienen nada que ver con un mejor desempeño general de sostenibilidad. Solo la calidad de la información está relacionada con los comportamientos de sostenibilidad, porque las instituciones de microfinanzas clasificadas como de mayor calidad de divulgación pueden producir mejores resultados de sostenibilidad social. 


\section{METODOLOGÍA}

La finalidad de esta investigación consistió en lograr el acercamiento y la cooperación entre la Facultad de Contabilidad y Auditoría, Universidad Pequeñas y Medianas Empresas, y en última instancia obtener la cooperación del sector industrial, para que puedan realizar la aplicación de los tributos, con los procedimientos correctos, sin los prejuicios u obstáculos que antes existían.

La teoría y la práctica proporcionaron sugerencias significativas para el desarrollo fundamental de la eficiencia administrativa en las pequeñas y medianas empresas. Por tal razón, los gobiernos deben considerar que solo los ajustes a los indicadores cuantitativos de desempeño dentro de un rango (como el porcentaje de prestatarios) pueden afectar negativamente la misión de desarrollo de las instituciones tributarias como el ministerio de industrias y productividad (Mipro).

Para el desarrollo de esta investigación, se recurrió al uso de encuestas para recopilar información referente a la tributación y a la eficiencia administrativa de las pequeñas y medianas empresas registradas en el Mipro del cantón Manta, provincia de Manabí.

A continuación, se muestra el esquema de encuesta con un total de 15 preguntas realizadas al sector de las pymes y un total de 5 preguntas realizadas a la comunidad universitaria.

Tabla 1. Check list de la encuesta al sector de las Pymes de Manta

\begin{tabular}{|l|l|}
\hline No. & \multicolumn{1}{c|}{ PREGUNTAS REALIZADAS } \\
\hline 1 & Su empresa actualmente está inscrita en el MIPRO. \\
\hline 2 & Si su empresa está inscrita en el MIPRO cómo está registrada. \\
\hline 3 & En qué régimen impositivo usted está registrado ante el SRI. \\
\hline 4 & Está su empresa obligada a llevar contabilidad. \\
\hline 5 & Mantiene usted facturación electrónica. \\
\hline 6 & $\begin{array}{l}\text { Cuáles de las siguientes declaraciones y anexos está usted obligado a presentar } \\
\text { ante el SRI }\end{array}$ \\
\hline 7 & $\begin{array}{l}\text { En el año anterior, ¿Cuáles de los siguientes medios utilizó su empresa para } \\
\text { obtener información sobre sus obligaciones tributarias, }\end{array}$ \\
\hline
\end{tabular}




\begin{tabular}{|c|l|}
\hline 8 & $\begin{array}{l}\text { Durante el presente año, ¿Su empresa realizó alguna de las siguientes actividades de } \\
\text { información y/o capacitación sobre sus obligaciones tributarias? ¿Cuánto tiempo le } \\
\text { dedicó a cada una de ellas en TOTAL. }\end{array}$ \\
\hline 9 & $\begin{array}{l}\text { En el presente año, ¿Su empresa utilizó la página web del SRI para cumplir con sus } \\
\text { obligaciones tributarias y/o realizar algún trámite. }\end{array}$ \\
\hline 10 & Qué trámites realizó su empresa durante el año. \\
\hline 11 & $\begin{array}{l}\text { Cuántotiempo MENSUAL (horas) ha dedicado su empresa a conectarse ala página } \\
\text { web del SRI. }\end{array}$ \\
\hline 12 & $\begin{array}{l}\text { Qué medios utilizan habitualmente en su empresa para el registro contable de las } \\
\text { operaciones de su negocio. }\end{array}$ \\
\hline 13 & $\begin{array}{l}\text { Cuánto es el valor estimado MENSUAL que su empresa gasta en los siguientes } \\
\text { ítems: desde la pregunta 13 a la 17. }\end{array}$ \\
\hline 14 & $\begin{array}{l}\text { Cuántas personas trabajan en su empresa para cumplir mensualmente con las } \\
\text { obligaciones tributarias. }\end{array}$ \\
\hline 15 & Su empresa tuvo que hacer alguna de las siguientes acciones post declaración. \\
\hline
\end{tabular}

Tabla 2. Encuestas realizadas a estudiantes del sexto al décimo nivel de la Facultad de Contabilidad y Auditoría.

\begin{tabular}{|c|l|}
\hline No. & \multicolumn{1}{c|}{ PREGUNTAS REALIZADAS } \\
\hline 1 & Su empresa actualmente está inscrita en el MIPRO. \\
\hline 2 & Si su empresa está inscrita en el MIPRO cómo está registrada. \\
\hline 3 & En qué régimen impositivo usted está registrado ante el SRI. \\
\hline 4 & Está su empresa obligada a llevar contabilidad. \\
\hline 5 & Mantiene usted facturación electrónica. \\
\hline
\end{tabular}

\section{RESULTADOS Y DISCUSIÓN}

En el contexto de paradigmas tecnológicos innovadores, la forma y el contenido de la administración tributaria está cambiando constantemente. Autores como Ngoasong, MZ y Kimbu, AN (2016) afirman que las instituciones de microfinanzas apoyan significativamente las empresas impulsadas en gran medida por el desarrollo fiscal al proporcionar microcréditos y oportunidades de desarrollo a las pequeñas y medianas empresas, abundantes en los países en desarrollo, así como a proyectos comunitarios y actividades de divulgación que a su vez promuevan las actividades comerciales de las instituciones microfinancieras informales. 
Tabla 3. Resultados de las encuestas realizadas a varias Pymes de Manta.

EFICIENCIA ADMINISTRATIVA EN LA PYME'S DEL CANTÓN MANTA

\begin{tabular}{|c|c|c|}
\hline \multicolumn{3}{|c|}{ EFICIENCIA ADMINISTRATIVA EN LA PYME'S DEL CANTÓN MANTA } \\
\hline No. & PREGUNTAS REALIZADAS & RESPUESTAS \\
\hline 1 & $\begin{array}{l}\text { Su empresa actualmente está } \\
\text { inscrita en el MIPRO. }\end{array}$ & $52 \% \mathrm{SI}$ Y $48 \% \mathrm{NO}$ \\
\hline 2 & $\begin{array}{l}\text { Si su empresa está inscrita en el } \\
\text { MIPRO cómo está registrada. }\end{array}$ & $56 \%$ Micro, $29 \%$ pequeña y $15 \%$ mediana. \\
\hline 3 & $\begin{array}{l}\text { En qué régimen impositivo usted } \\
\text { está registrado ante el SRI. }\end{array}$ & $\begin{array}{l}\text { El 58,18\% de las PYMES está registrada en el } \\
\text { Régimen Impositivo General, 20,91\% Régimen } \\
\text { Impositivo Simplificado Ecuatoriano y } 20,91 \% \text { al } \\
\text { Régimen de la Micro empresa. }\end{array}$ \\
\hline 4 & $\begin{array}{l}\text { Está su empresa obligada a } \\
\text { llevar contabilidad. }\end{array}$ & $\begin{array}{l}\text { El } 54,45 \% \text { PYMES obligadas a llevar contabilidad y } \\
\text { el } 54,55 \% \text { no están obligadas a llevar contabilidad. }\end{array}$ \\
\hline 5 & $\begin{array}{l}\text { Mantiene usted facturación } \\
\text { electrónica. }\end{array}$ & $\begin{array}{l}34,55 \% \text { de las PYMES si está emitiendo facturación } \\
\text { electrónica, mientras que la mayoría, es decir el } \\
65,45 \text { no lo hace. }\end{array}$ \\
\hline 6 & $\begin{array}{l}\text { Cuáles de las siguientes } \\
\text { declaraciones y anexos está } \\
\text { usted obligado a presentar ante } \\
\text { el SRI. }\end{array}$ & $\begin{array}{l}71,82 \% \text { de las PYMES presenta declaraciones del } \\
\text { IVA mensual, el } 27 \% \text { lo hace de manera semestral. } \\
\text { Así mismo el } 30 \% \text { realiza retenciones y el } 13 \% \text { no } \\
\text { presentan anexos. }\end{array}$ \\
\hline 7 & $\begin{array}{l}\text { En el año anterior, ¿Cuáles de los } \\
\text { siguientes medios utilizó su } \\
\text { empresa para obtener } \\
\text { información sobre r sus } \\
\text { obligaciones tributarias. }\end{array}$ & $\begin{array}{l}\text { El } 60 \% \text { de las PYMES utiliza la página WEB del SRI } \\
\text { y apenas el } 24,55 \% \text { va a sus oficinas, mientras que } \\
\text { un número muy inferior de ellas llama al SRI o lo } \\
\text { hace a través de un asesor externo. }\end{array}$ \\
\hline 8 & $\begin{array}{l}\text { Durante el presente año, ¿Su } \\
\text { empresa realizó alguna de las } \\
\text { siguientes actividades de } \\
\text { información y/o capacitación sobre } \\
\text { sus obligaciones tributarias? } \\
\text { ¿Cuánto tiempo le dedicó a cada } \\
\text { una de ellas en TOTAL. }\end{array}$ & $\begin{array}{l}\text { Las empresas en su gran mayoría han realizado } \\
\text { capacitación y consultas al SRI, de manera virtual, } \\
\text { lo cual evidencia que falta mayor información de } \\
\text { parte de esta institución, ya que las PYMES se ven } \\
\text { obligadas a capacitar por cuenta propia a su } \\
\text { personal, lo cual incide en sus costos } \\
\text { administrativos. }\end{array}$ \\
\hline 9 & $\begin{array}{l}\text { En el presente año, ¿Su empresa } \\
\text { utilizó la página web del SRI para } \\
\text { cumplir con sus obligaciones } \\
\text { tributarias y/o realizar algún } \\
\text { trámite. }\end{array}$ & $\begin{array}{l}\text { El } 80,91 \% \text { de las PYMES cumple con sus } \\
\text { obligaciones tributarias de manera virtual y para } \\
\text { realizar algún trámite, mientras que sólo el } 19,09 \% \\
\text { no lo hace. }\end{array}$ \\
\hline 10 & $\begin{array}{l}\text { ¿Qué trámites realizó su empresa } \\
\text { durante el año? }\end{array}$ & $\begin{array}{l}\text { El } 71,82 \% \text { de las PYMES realizó trámites de } \\
\text { declaración y pago del IVA y el } 38 \% \text { realiza siempre } \\
\text { consultas sobre su situación tributaria, actualización } \\
\text { del RUC y por sus propias declaraciones de } \\
\text { impuestos. }\end{array}$ \\
\hline 11 & $\begin{array}{l}\text { ¿Cuánto tiempo MENSUAL } \\
\text { (horas) ha dedicado su empresa } \\
\text { a conectarse a la página web del } \\
\text { SRI? }\end{array}$ & $\begin{array}{l}\text { Las PYMES ocupan gran cantidad de tiempo para } \\
\text { conectarse a la página del SRI; lo cual evidencia una } \\
\text { lentitud de la plataforma de esta institución y que } \\
\text { genera costos adicionales al empresario. }\end{array}$ \\
\hline
\end{tabular}




\begin{tabular}{|c|c|c|}
\hline 12 & $\begin{array}{l}\text { ¿Qué medios utilizan } \\
\text { habitualmente en su empresa para } \\
\text { el registro contable de las } \\
\text { operaciones de sunegocio? }\end{array}$ & $\begin{array}{l}\text { El } 39,39 \% \text { de las PYMES aún realiza sus registros } \\
\text { contables en libros físicos, mientras que el } 48 \% \\
\text { aproximadamente lo hace en programas } \\
\text { computarizados propios u otorgados por el SRI. }\end{array}$ \\
\hline 13 & $\begin{array}{l}\text { ¿Cuánto es el valor estimado } \\
\text { MENSUAL que su empresa } \\
\text { gasta en los siguientes ítems: } \\
\text { desde la pregunta } 13 \text { a la } 17 ?\end{array}$ & $\begin{array}{l}\text { Para cumplir con sus obligaciones tributarias, las } \\
\text { PYMES, normalmente gastan: } \\
\text { a).- } 100 \text { dólares en materiales de apoyo. } \\
\text { b.- De entre } 50 \text { a } 150 \text { dólares en trámites y } \\
\text { movilización. } \\
\text { c.- Entre } 50 \text { a } 180 \text { dólares en la compra o utilización } \\
\text { de programas informáticos para el registro de sus } \\
\text { actividades. } \\
\text { d). - Entre } 10 \text { a } 70 \text { dólares, en costos asociados a la } \\
\text { facturación electrónica. } \\
\text { e).- Entre } 10 \text { a } 50 \text { dólares mensuales, en cualquier } \\
\text { gasto relacionado al cumplimiento de sus } \\
\text { obligaciones tributarias. }\end{array}$ \\
\hline 14 & $\begin{array}{l}\text { ¿Cuántas personas trabajan en } \\
\text { su empresa para cumplir } \\
\text { mensualmente con las } \\
\text { obligaciones tributarias? }\end{array}$ & Entre 2 a 6 personas. \\
\hline 15 & $\begin{array}{l}\text { ¿Su empresa tuvo que hacer } \\
\text { alguna de las siguientes } \\
\text { acciones post declaración? }\end{array}$ & $\begin{array}{l}\text { La gran mayoría de las PYMES ocupa mucho } \\
\text { tiempo en varias acciones post declaraciones de } \\
\text { impuestos, lo cual se transcribe en costos } \\
\text { administrativos. }\end{array}$ \\
\hline
\end{tabular}

\section{- Análisis y discusión.}

PREGUNTA 1.- Su empresa actualmente está inscrita en el MIPRO?

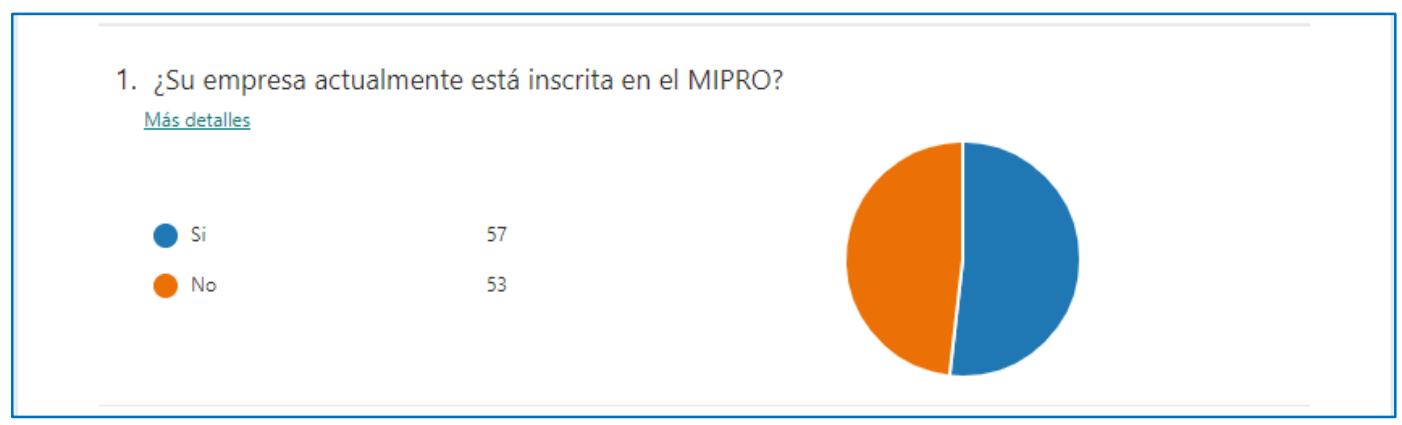

Los resultados de la pregunta $N^{\circ} 1$, de la encuesta realizada, establecen que el $52 \%$ de las empresas se encuentra inscrita en el MIPRO, mientras que el $48 \%$ no lo está, lo cual nos da una idea general de que más de la mitad, puede tener mayores beneficios, gracias a su registro en una institución del estado. 
PREGUNTA 2.- Si su empresa está inscrita en el MIPRO cómo está registrada?

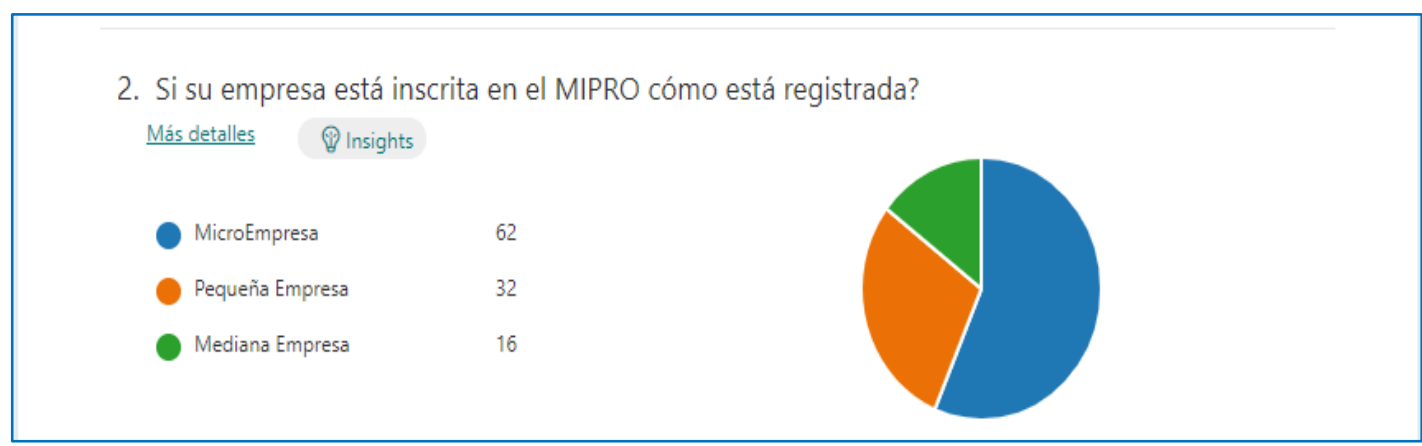

Los resultados de la pregunta $\mathrm{N}^{\circ} 2$, demuestran que el $56 \%$ de las empresas registradas en el MIPRO, se encuentran clasificadas como Microempresas, es decir tienen ingreso menores a 100.000 dólares y manejan un personal de máximo 9 trabajadores, que el 29\% está clasificadas como Pequeña Empresa, es decir tienen ingresos de entre 100.001 dólares hasta 1 millón de dólares y ocupan mano de obra de hasta 49 trabajadores, mientras que el 15\% está clasificadas como Mediana Empresa; es decir, que tienen ingresos de entre 1 millón a 5 millones de dólares y manejan un personal de entre 55 a 199 trabajadores.

PREGUNTA 3.- En qué régimen impositivo usted está registrado ante el SRI?

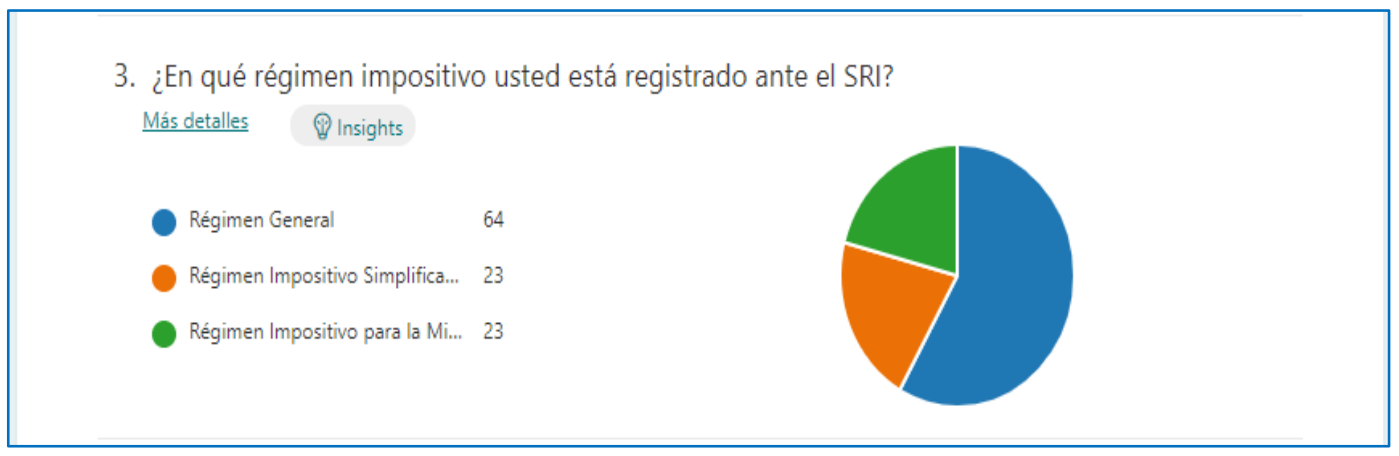

Los resultados de la pregunta $^{\circ} 3$, nos indican que el $58,18 \%$ de las empresas encuestadas, están registradas en el SRI en el Régimen Impositivo General, el 20,91\% en el Régimen Impositivo Simplificado Ecuatoriano RISE y el otro 20,91\% en el nuevo Régimen para la Microempresa. 
PREGUNTA 4.- Está su empresa obligada a llevar contabilidad?

4. ¿Está su empresa obligada a llevar contabilidad?

Más detalles \& Insights

Si 50

No

60

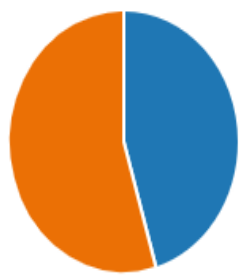

Los resultados de la pregunta $N^{\circ} 4$, nos indican que el $54,45 \%$ de las empresas encuestadas, si son obligadas a llevar contabilidad, es decir que operan con un capital superior a $\$ 180.000,00$, que sus ingresos anuales son superiores a $\$ 300.000,00$ o que sus costos o gastos anuales, son superiores a $\$ 240.000,00$; mientras que el 54,55\% no están obligadas a llevar contabilidad.

PREGUNTA 5.- Mantiene usted facturación electrónica?

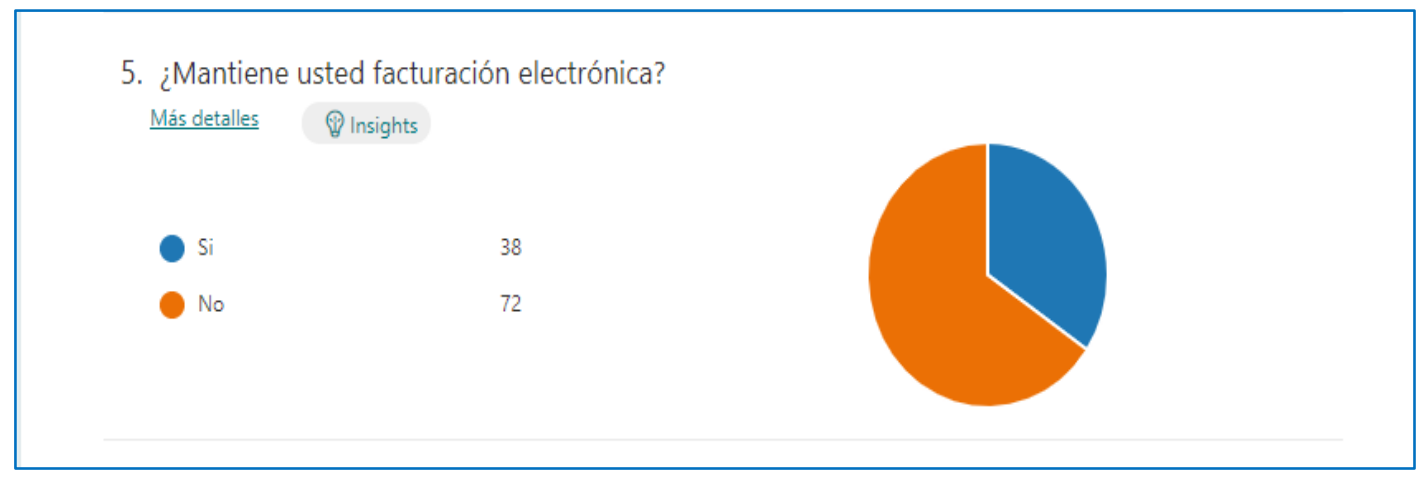

Los resultados de la pregunta $N^{\circ} 5$, identifican a un sector empresarial que en un 34,55\% si está emitiendo facturación electrónica, mientras que la mayoría, es decir el 65,45\% no lo está, lo cual evidencia quizás una ausencia de automatización de los registros contables y de facturación, por una buena parte del sector empresarial de Manta. 
PREGUNTA 6.- Cuáles de las siguientes declaraciones y anexos está usted obligado a presentar ante el SRI?

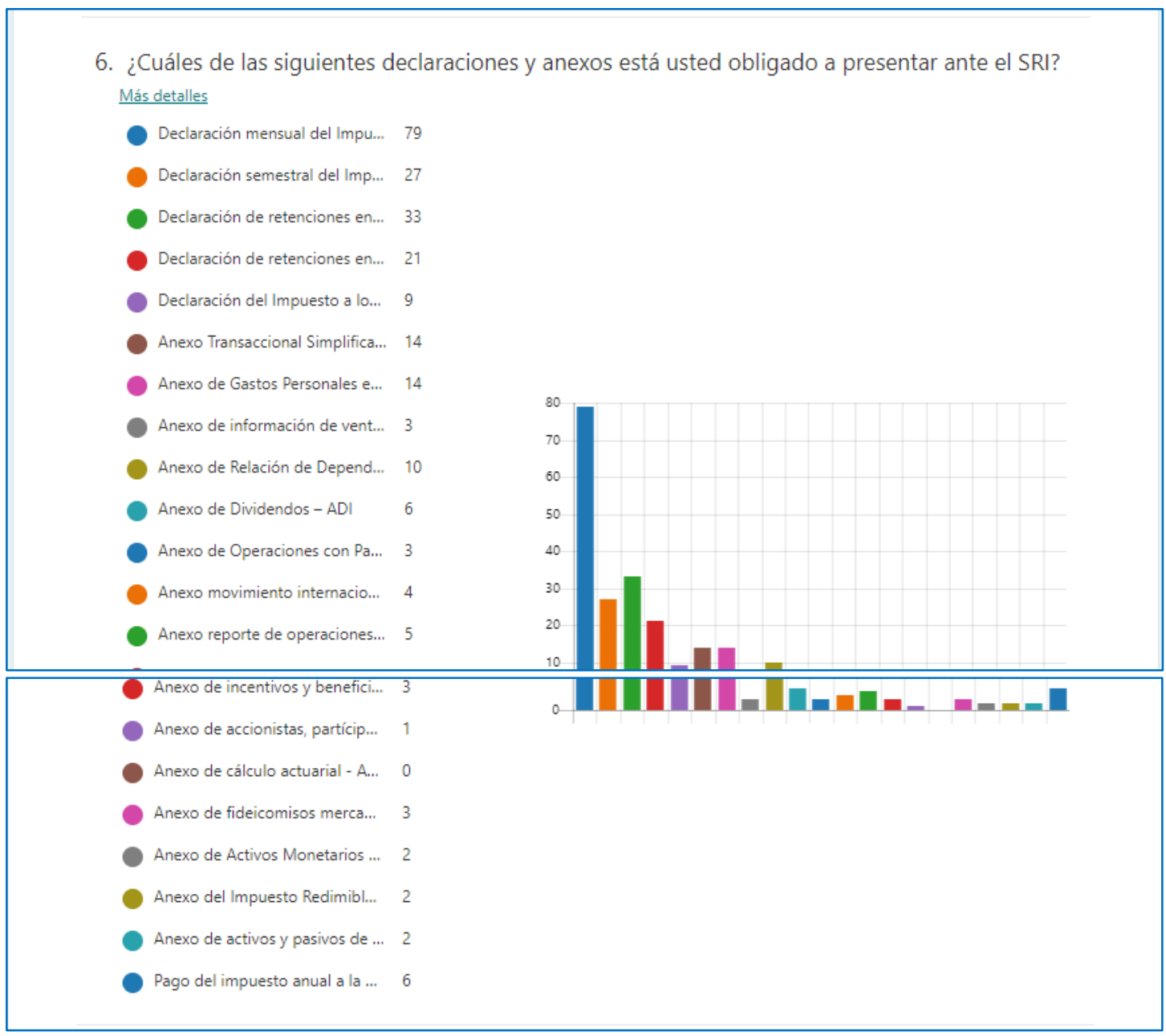

Los resultados de la pregunta $N^{\circ} 6$, demuestran que el sector empresarial de las PYMES de Manta, realiza en su gran mayoría 71,82\% las declaraciones de IVA de manera mensual y sólo el $27 \%$ lo hace de manera semestral, lo cual es compatible con el hecho de que el 58\% están registradas en el SRI al Régimen Impositivo General. Así mismo se puede notar que sólo el 30\% realiza retenciones y una gran minoría que no llega al 13\% de todas las empresas encuestadas, no presentan anexos, esto se debe seguramente a que sólo el $54 \%$ están obligadas a llevar contabilidad. 
PREGUNTA 7.- En el año anterior, ¿Cuáles de los siguientes medios utilizó su empresa para obtener información sobre sus obligaciones tributarias?

7. En el año anterior, ¿Cuáles de los siguientes medios utilizó su empresa para obtener información sobre sus obligaciones tributarias?

Más detalles insights

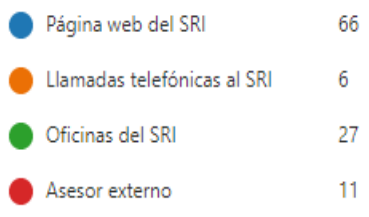

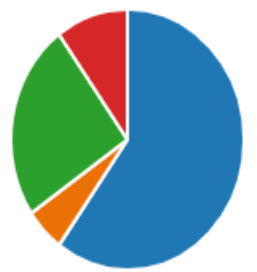

Los resultados de la pregunta $N^{\circ} 7$, nos indica que el sector empresarial de la PYMES de Manta, si mantiene sistema de internet, puesto que el $60 \%$ utiliza la página WEB del SRI, para consultar y apenas $24,55 \%$ va a sus oficinas, mientras que un número muy inferior de ellas llama al SRI o lo hace a través de un asesor externo.

PREGUNTA 8.- Durante el presente año, ¿Su empresa realizó alguna de las siguientes actividades de información y/o capacitación sobre sus obligaciones tributarias? ¿Cuánto tiempo le dedicó a cada una de ellas en TOTAL?

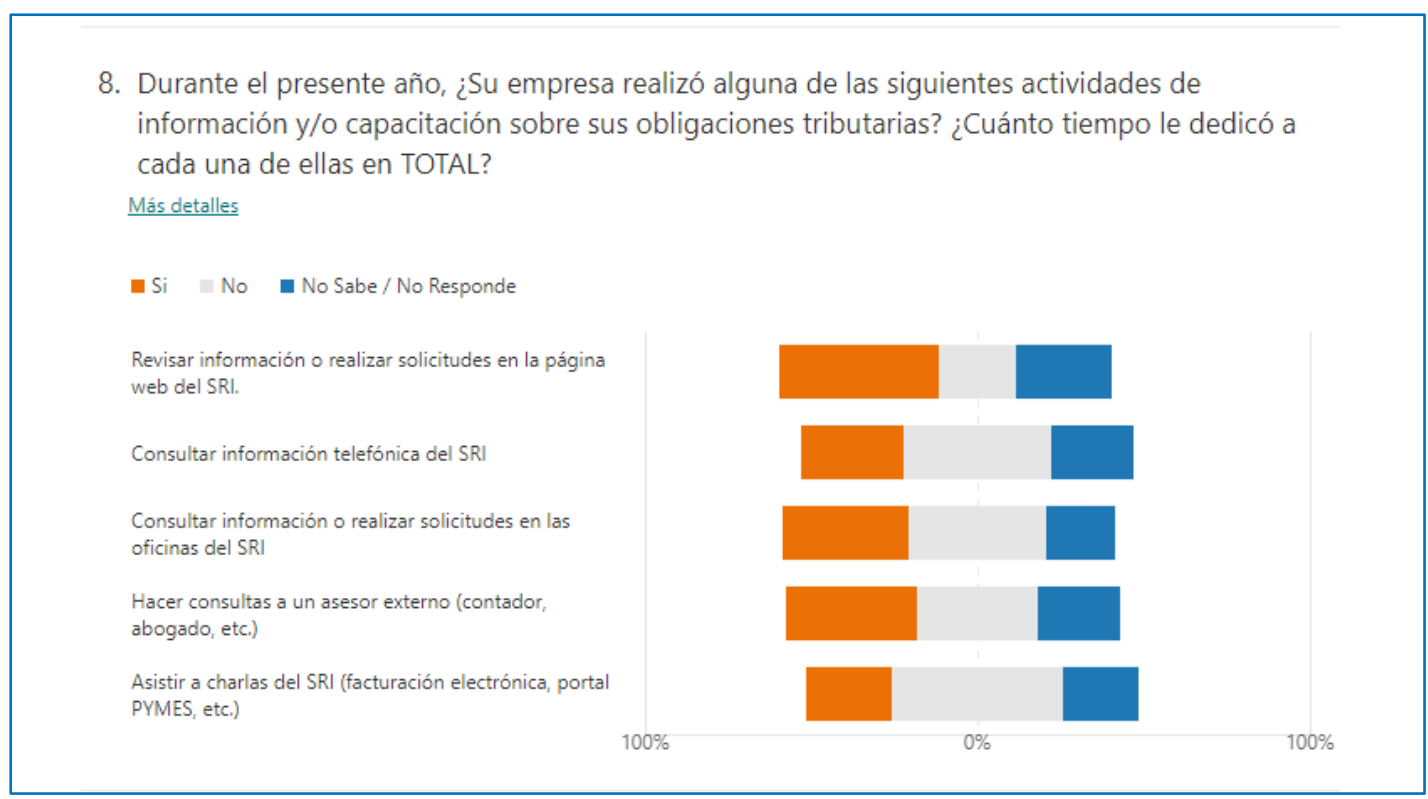

Los resultados de la pregunta $\mathrm{N}^{\circ} 8$ de la encuesta, al sector de la Micro y Mediana Empresa de Manta, es congruente con los resultados de la pregunta anterior, puesto que las empresas en su gran mayoría han realizado capacitación y consultas al SRI, de manera virtual, lo cual evidencia que falta mayor información de parte de esta institución, ya que las PYMES se ven obligadas a 
capacitar a su personal de manera permanente, lo cual incide en el aumento de sus costos administrativos.

PREGUNTA 9.-Enelpresenteaño, ¿SuempresautilizólapáginawebdelSRIparacumplir con sus obligaciones tributarias y/o realizar algún trámite.?

9. En el presente año, ¿Su empresa utilizó la página web del SRI para cumplir con sus obligaciones tributarias y/o realizar algún trámite.?

Más detalles

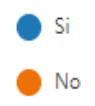

89

21

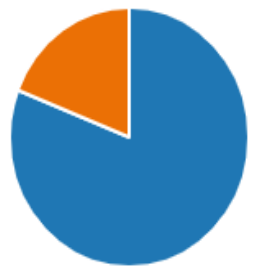

Los resultados de la pregunta $\mathrm{N}^{\circ}$ 9, de esta encuesta sector de las PYMES de Manta, sigue siendo compatible con las 2 respuestas anteriores, puesto que el $80,91 \%$ de ellas; cumple con sus obligaciones tributarias de manera virtual y realizar algún trámite, mientras que sólo el 19,09\% no lo hace.

PREGUNTA 10.- ¿Qué trámites realizó su empresa durante el año?

10. ¿Qué trámites realizó su empresa durante el año? (Marque lo que corresponda) Más detalles

Consulta de situación tributaria 42

Declaración y/o pago del IVA 79

Obtención, recuperación o ca... 17

Actualización del RUC

Emisión documentos tributari... 15

Declaración y/o pago del Imp... 43

NS/NR

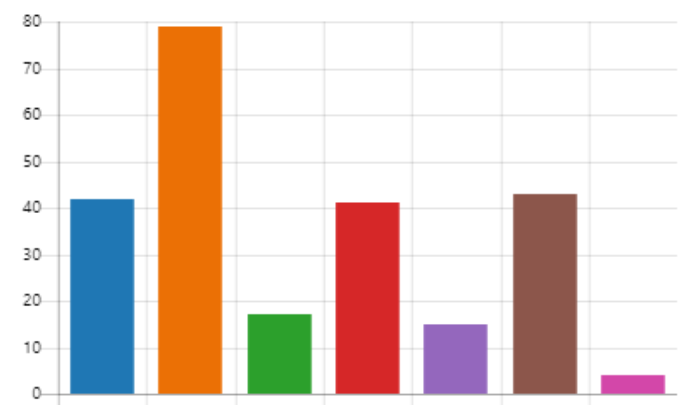

Los resultados de la pregunta $\mathrm{N}^{\circ} 10$, son congruentes con las 3 últimas preguntas y nos permiten apreciar que el sector de las PYMES de Manta en un $71,82 \%$ realizó trámites de declaración y pago del IVA y que aproximadamente el 38\% realiza siempre consultas sobre su situación tributaria, actualización del RUC y por sus propias declaraciones de impuestos. 
PREGUNTA 11.- Cuánto tiempo MENSUAL (horas) ha dedicado su empresa a conectarse a la página web delSRI?

11. ¿Cuánto tiempo MENSUAL (horas) ha dedicado su empresa a conectarse a la página web del SRI?

Más detalles

Los resultados de la pregunta $N^{\circ} 11$, son contundentes respecto de la gran cantidad de tiempo que ocupan los micro empresarios, a conectarse a la página del SRI; lo cual evidencia que la lentitud de la plataforma de esta institución, genera muchos costos adicionales al empresario.

PREGUNTA 12.- Qué medios utilizan habitualmente en su empresa para el registro contable de las operaciones de su negocio? (Marque una o más opciones sicorresponde).

12. ¿Qué medios utilizan habitualmente en su empresa para el registro contable de las operaciones de su negocio? (Marque una o más opciones si corresponde). Más detalles

Registros o libros físicos (Papel). 52

Registros en programas comp... 22

Registros en programas comp... 41

NS /NR.

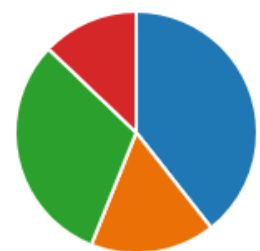

Los resultados de la pregunta $\mathrm{N}^{\circ} 12$, nos demuestra que el sector empresarial encuestado, en el 39,39\% aún realiza sus registros contables en libros físicos, mientras que el $48 \%$ aproximadamente lo hace en programas computarizados propios u otorgados por el SRI.

PREGUNTA 13.- Cuánto es el valor estimado MENSUAL que su empresa gasta en los siguientes

13. ¿Cuánto es el valor estimado MENSUAL que su empresa gasta en lo siguiente: Compra de formularios y materiales, como libros, boletas, facturas, guías de despacho, etc. Más detalles 
Los resultados de la pregunta $\mathrm{N}^{\circ} 13$ de esta encuesta, indican que el sector empresarial encuestado, gasta más de 100 dólares mensuales en materiales de apoyo para poder cumplir con sus obligaciones tributarias.

PREGUNTA 14.- Cuánto es el valor estimado MENSUAL que su empresa gasta en los siguientes ítems:

14. ¿Cuánto es el valor estimado MENSUAL que su empresa gasta en lo siguiente: Movilización para hacer trámites relacionados con impuestos (visitar al contador, acudir al SRI).

Más detalles

Los resultados de la pregunta $N^{\circ} 14$ de esta encuesta, nos indica que el sector empresarial encuestado, gasta entre 50 a 150 dólares mensuales en trámites y movilización para poder cumplir con sus obligaciones tributarias.

PREGUNTA 15.- Cuánto es el valor estimado MENSUAL que su empresa gasta en los siguientes ítems:

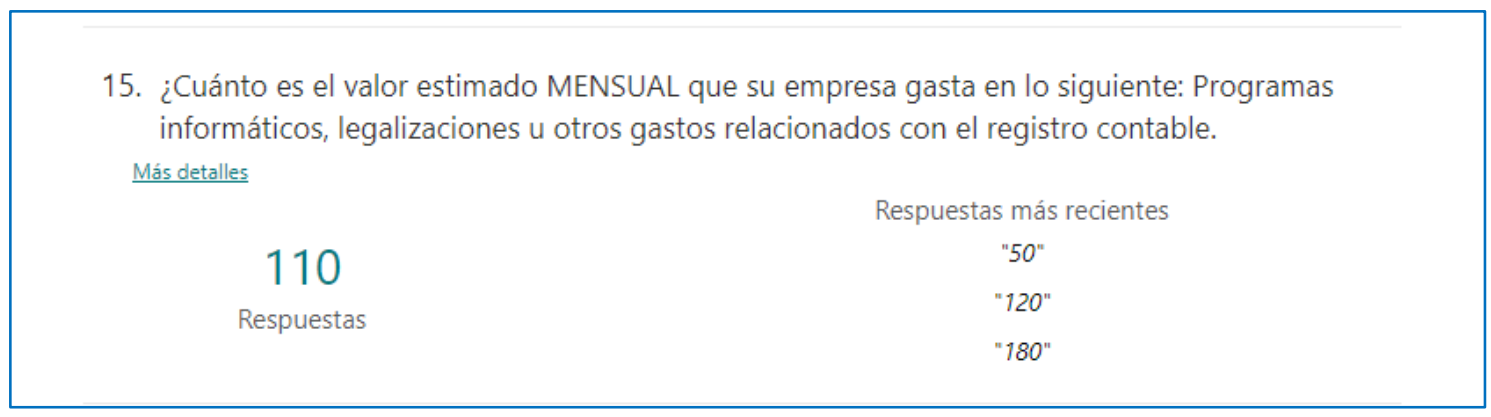

Los resultados de la pregunta $N^{\circ} 15$ de esta encuesta, nos indica que el sector empresarial encuestado, gasta entre 50 a 180 dólares mensuales en la compra o utilización de programas informáticos para el registro de sus actividades, para así poder cumplir con sus obligaciones tributarias. 


\section{Resultados de las encuestas realizadas a estudiantes del sexto al décimo nivel de la facultad de contabilidad y auditoría.}

PREGUNTA 1.- Se encuentra usted actualmente laborando.

1. ¿Se encuentra usted actualmente laborando?

Más detalles

Insights
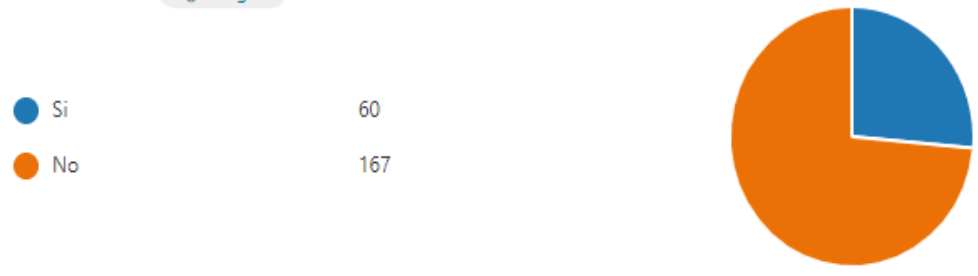

Los resultados de la pregunta $\mathrm{N}^{\circ} 1$ de la encuesta realizada, establecen que el $70 \%$ de los estudiantes de la Facultad de Contabilidad y Auditoría, no se encuentra laborando actualmente y que sólo el 30\% lo está haciendo.

Esta situación, denota que quizás por efectos de la pandemia las empresas no están contratando personal contable, debido a que muchas de ellas cerraron sus negocios o en su defecto, a los estudiantes, les falta mayor práctica y experiencia laboral, para que sean contratados.

PREGUNTA 2.- Cuando usted solicita trabajo, que requisito mayormente le exigen para contratarlo.

2. ¿Cuándo usted solicita trabajo, que requisito mayormente le exigen para contratarlo? Más detalles
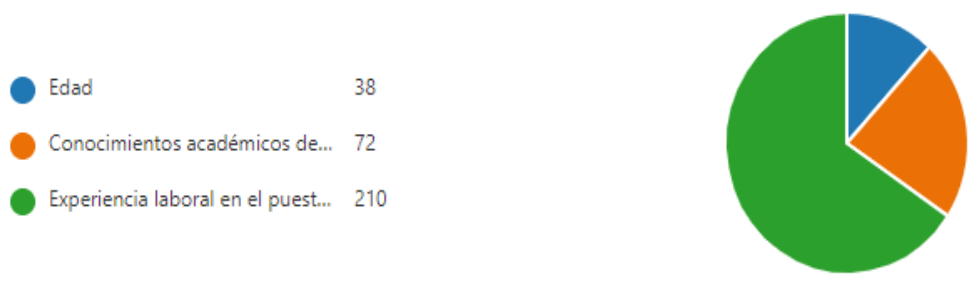

Los resultados de la pregunta $\mathrm{N}^{\circ} 2$ de la encuesta realizada, establecen que el $66 \%$ de los estudiantes de la Facultad de Contabilidad y Auditoría, cuando han acudido a solicitar un trabajo, le han requerido de experiencia laboral como requisito principal, mientras que al $23 \%$ de los estudiantes encuestados, le han solicitado que tengan conocimientos académicos dentro del campo laboral; así 
mismo sólo el $11 \%$ de los estudiantes, han manifestado que no lo contratan en las empresas, por su edad.

Los resultados de este análisis demuestran que, es necesario buscar mecanismos que le permitan a los estudiantes de la Facultad de Contabilidad y Auditoría, adquirir paralelamente a su preparación profesional dentro de la Universidad, la suficiente experiencia laboral en el área contable, para que llegue a ser más competitivo y la profesión sea mayormente demandada por los empresarios.

Por otro lado, podemos evidenciar, que el conocimiento académico que tengan los estudiantes de la Facultad de Contabilidad y Auditoría es secundario para el empresario, ya que éstos en la mayoría de los casos, siempre busca personal con experiencia y desde luego que tengan conocimientos en el área contable.

Así mismo, es evidente que la edad de los estudiantes de la Facultad de Contabilidad y Auditoría, no es importante para los empresarios a la hora de contratarlos.

PREGUNTA 3.- Le gustaría adquirir mayor experiencia práctica diréctamente en el campo laboral.

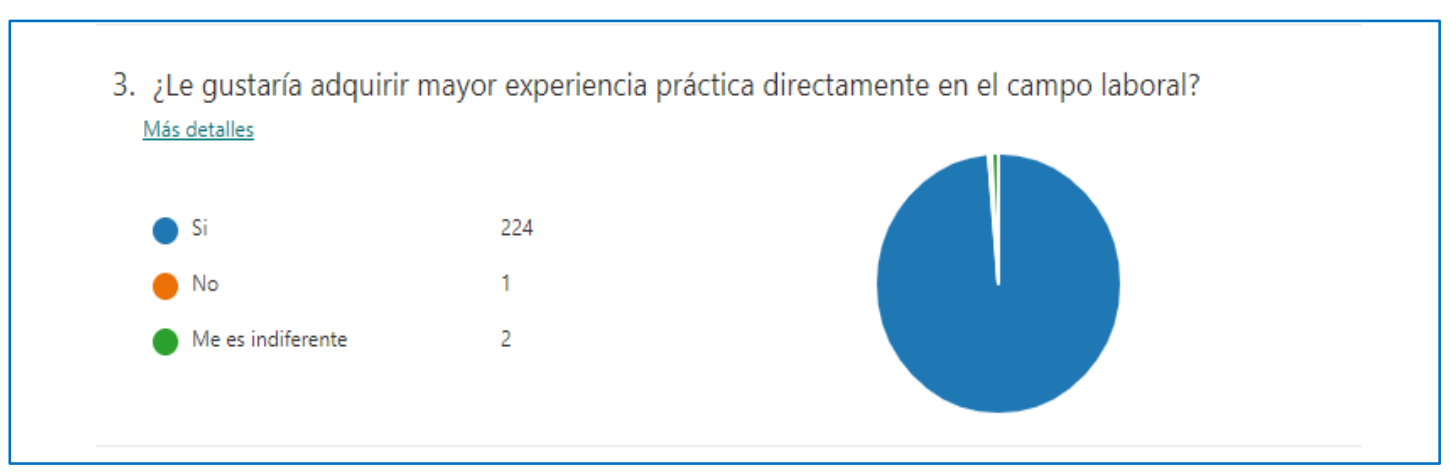

Los resultados de la pregunta $N^{\circ} 3$ de la encuesta realizada, establecen que el 99\% de los estudiantes de la Facultad de Contabilidad y Auditoría, necesitan imperativamente adquirir conocimientos en la práctica laboral del día a día, ya que es la única manera que ellos consideran que tendrían la posibilidad de adquirir experiencia práctica laboral dentro de su campo del conocimiento, que a su vez, les abra las puertas para la inserción laboral y las oportunidades para poder desarrollar su potencial intelectual y sus capacidades cognitivas, adquiridas dentro de la Universidad y de manera particular, en la Facultad de 
Contabilidad y Auditoría, así mismo podemos analizar que sólo a 1 estudiante no le gustaría adquirir experiencia laboral y que apenas a 2 estudiantes le es indiferente tener experiencia en su campo profesional.

PREGUNTA 4.- Le gustaría realizar sus prácticas laborales y de vinculación, en una propia microempresa, anexa a la Facultad de Auditoría.

4. ¿Le gustaría realizar sus prácticas laborales y de vinculación, en una propia micro empresa, anexa a la Facultad de Auditoría? Más detalles
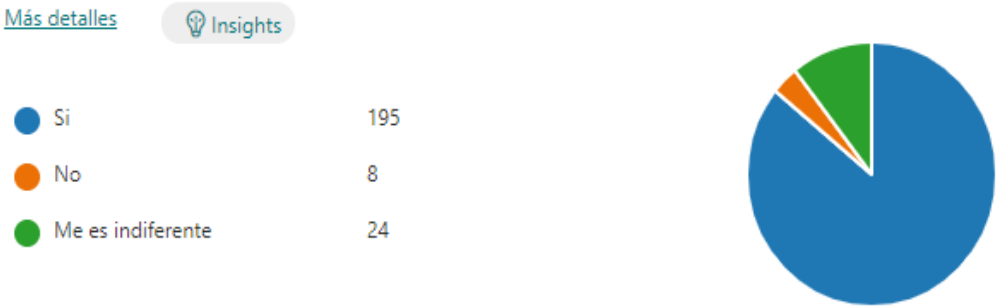

Los resultados de la pregunta $N^{\circ} 4$ de la encuesta realizada, establecen que al $86 \%$ de los estudiantes de la Facultad de Contabilidad y Auditoría, si les gustaría realizar sus prácticas laborales y de vinculación, en una microempresa propia de la Facultad de Contabilidad y Auditoría, así mismo al 3\% de los estudiantes no le gustaría hacerlo y al $11 \%$ les es indiferente si realizan o no sus prácticas laborales en una microempresa anexa a la Facultad de Contabilidad y Auditoría.

Este análisis demuestra que, en su gran mayoría, los estudiantes de la Facultad de Contabilidad y Auditoría ven en esta posibilidad de crear una microempresa anexa a su Facultad, la posibilidad de adquirir el conocimiento y la práctica laboral que ellos necesitan, para poder ser contratados por el sector empresarial.

PREGUNTA 5.- Le gustaría que la Universidad y la Facultad de Contabilidad y Auditoría, le certifiquen su experiencia laboral adquirida.

5. ¿Si su respuesta fue si en la pregunta anterior, le gustaría que la Universidad y la Facultad de Contabilidad y Auditoría, le certifique su experiencia laboral adquirida? Más detalles (1) Insights

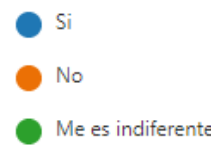

191

3

1

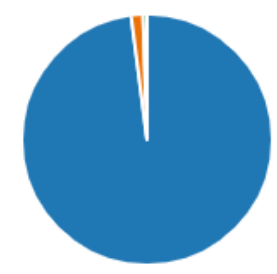


Los resultados de la pregunta $N^{\circ} 5$ de la encuesta realizada, establecen que al 98\% de los estudiantes de la Facultad de Contabilidad y Auditoría, si les gustaría que la Universidad Laica Eloy Alfaro de Manabí y la Facultad de Contabilidad y Auditoría, le certifiquen que ha realizado sus prácticas laborales dentro de la empresa anexa a su Facultad, mientras que sólo al $2 \%$ de los estudiantes no le importa.

Estas respuestas de los estudiantes demuestran que es necesario y urgente que la Universidad y la Facultad de Contabilidad y Auditoría, se preocupen por entregar al sector laboral, no sólo a un profesional académicamente formado, sino que además posea la suficiente experiencia laboral, que bien la podría adquirir dentro de la misma Universidad, a través de una microempresa anexa, donde los estudiantes, pongan en práctica los conocimientos adquiridos en el aula de clases.

\section{CONCLUSIONES}

Las pequeñas empresas son aquellas las que tienen cierto problema en cuanto a la aplicación de los tributos, porque muchas veces ellos no tienen el ingreso esperado en el año y sin embargo debe declarar impuestos como actualmente que al regirse por el régimen de microempresa debe declarar de forma semestral y la declaración del $2 \%$ sobre los ingresos, porcentaje por el cual muchos contribuyentes no estaban de acuerdo y que obviamente afecta a la liquidez financiera del negocio independientemente de la actividad.

Las normas jurídicas que establecen los tributos y que todo sujeto pasivo específicamente las pymes deben acatar y cumplir es: la Ley Orgánica de Régimen Tributario Interno, el Reglamento de la LORTI, el código tributario y el Código Orgánico de la Producción, Comercio e Inversiones.

La administración tributaria a través del SRI, se encarga de dirigir la correcta declaración de los impuestos no solo a grandes empresas sino también a las pequeñas y mediana empresas, sin embargo, al fijarse de que este grupo es mayoritario y que de igual manera forma parte del desarrollo económico del país 
se han aplicado normas tributarias que los beneficien como es la exoneración de impuestos por 3 años.

Los resultados de la pregunta $N^{\circ} 4$ nos indican que el 54,45\% de las empresas encuestadas, si son obligadas a llevar contabilidad, es decir que operan con un capital superior a $\$ 180.000,00$, que sus ingresos anuales son superiores a $\$ 300.000,00$ o que sus costos o gastos anuales, son superiores a $\$ 240.000,00$; mientras que el $54,55 \%$ no están obligadas a llevar contabilidad. Dependiendo entonces de si están o no obligadas a llevar contabilidad, las PYMES tienen ya sus obligaciones establecidas en la ley y que ésta empresas atienden de manera técnica para evitar multas e intereses.

Dada la evidencia de la viabilidad de las reformas e innovaciones tributarias en el contexto europeo, a nivel latinoamericano, estas innovaciones podrían ayudar a los servicios de las instituciones microfinancieras a satisfacer las necesidades de muchos emprendedores, promoviendo el desarrollo productivo y mejorando las condiciones de vida en los países en desarrollo.

\section{REFERENCIAS}

Chávez, L. (2018). NIIF y tributación: desafíos y oportuniades para las administraciones tributarias.[IFRS and Taxation: challenges and opportunities for tax administrations]. Revista de administración tributaria CIATIAEATIIEF, (43), 6-26.

Mathieu-Bolh, N. (2017). Can tax reforms help achieve sustainable development? Resource and Energy Economics, 50, 135-163. doi:10.1016/j.reseneeco.2017.06.004

Ngoasong, M. Z., \& Kimbu, A. N. (2016). Informal microfinance institutions and development-led tourism entrepreneurship. Tourism Management, 52, 430-439. doi:10.1016/j.tourman.2015.07.012

Ocaña, J. C. L. (2017). Impacto de la gestión administrativa sobre la eficiencia de la actividad pesquera artesanal-Cantón Manta. Eca Sinergia, 8(2), 144157.

Oncebay Sacsara, K. Y. (2019). Financiamiento, rentabilidad y tributación de la micro y pequeña empresa del sector comercio, rubro plastiquería "Inversiones Ikiu” SRL-Ayacucho, 2019. 
Orellana, M. N., Gutiérrez, N. D., \& Feijoo, E. M. (2019). Los convenios de doble tributación en la recaudación de la administración tributaria del Ecuador. Revista ESPACIOS. ISSN: 0798, 1015.

Perilla, V. D. (2011). Calidad de la ley, técnica legislativa y eficiencia administrativa. Prolegómenos: Derechos y valores, 14(27), 147-163.

Viktor V. Ivanov, Natalia V. Pokrovskaia, Nadezhda A. Lvova, (2016). Tax Potential of a State: Development Factors. Proceedings of the International Conference on Accounting, Auditing, and Taxation (ICAAT) on December 08, 2016

Wunder Hachem, D., \& Gabardo, E. (2018). El principio constitucional de eficiencia administrativa: contenido normativo y consecuencias jurídicas de su violación. Cuestiones constitucionales, (39), 131-167. 